\title{
Hand Vien Recognotion Using Matlab
}

\author{
Rakesh Pandey, Akhilesh Mehta, Lalit singh Garia \\ ${ }^{I}$ (ECE M.Tech, BTKIT DWARAHAT / Uttarakhand technical university, India) \\ 2(ECE M.Tech, BTKIT DWARAHAT / Uttarakhand technical university, India) \\ 3(ECE , BTKIT DWARAHAT (India)
}

\begin{abstract}
Bold): Identification based on hand vein pattern is an interesting branch of biometric recognition that is enjoying increasingly attention among the researchers in the recent decade. However, despite the fact the most of the developed commercial systems for this purpose are expensive; their false acceptance ratios are not zero. Therefore, using them for identification purposes can be crucial if they mistakenly accept an imposter (even with a very low probability). The current work proposes a very low-cost hand vein pattern recognition system using a simple modified webcam. The system introduces a blob removal algorithm for enhancing the results of the segmentation and uses a modified version of Hausdorff distance for feature matching for the recognition purposes. Experimental results show that the system can achieve a zero false acceptance ratio while keeping the true acceptance rate in an acceptable level. (10 Italic)
\end{abstract}

Keywords (11Bold) - Pattern Recognition; Hand Vein; Biometrics; Infrared Imaging (10 Italic)

\section{INTRODUCTION (11 BOLD)}

Nowadays a very large number of identification systems exist that are based on different types of biometrics. This includes iris, fingerprint, retina, voice, face, palm and vascular pattern recognition. This last one is quite a new emerging technology in the biometric field, and has been gradually adopted over the world. The idea of using the hand vascular pattern as a biometric was first considered in the early 1990s but it wasn't until 1997 that a commercial product was developed. In 2000 it finally became popular when an application was created for personal identification based on the vein pattern on the back of the hand [1].

Since its introduction, hand vein pattern technology has expanded to fingers and palm based systems and was adopted in 2007 by the International Standard Organization (ISO) where the storage and transmission of vascular biometric images was standardized. The demand for secure identification systems has increased exponentially over the last ten years. These systems are required to be very reliable but also easy to use since their application is no longer restricted to high-security facilities. The advantages of hand vein pattern recognition are due to the fact that veins lie underneath the skin, which makes them easily accessible for the system but also hard to alter. In this perspective, the accessibility of vein pattern compared with other biometrics and its ease of use have made it a very interesting alternative for applications where a high level of security is required. It is also a good alternative to biometric systems that require physical contactin order to identify the individual, especially in environments such as hospitals where hygiene has high priority. An identification system should be fast, simple and secure and due to its desirable advantages, vein pattern technology is being considered into various authentication solutions for use in public places (access control, time and attendance, security, hospitals).The market for hand vascular pattern technology is growing rapidly and today it is an area of ongoing research that draws a lot of attention.

\section{THE PROPOSED ALgORITHM}

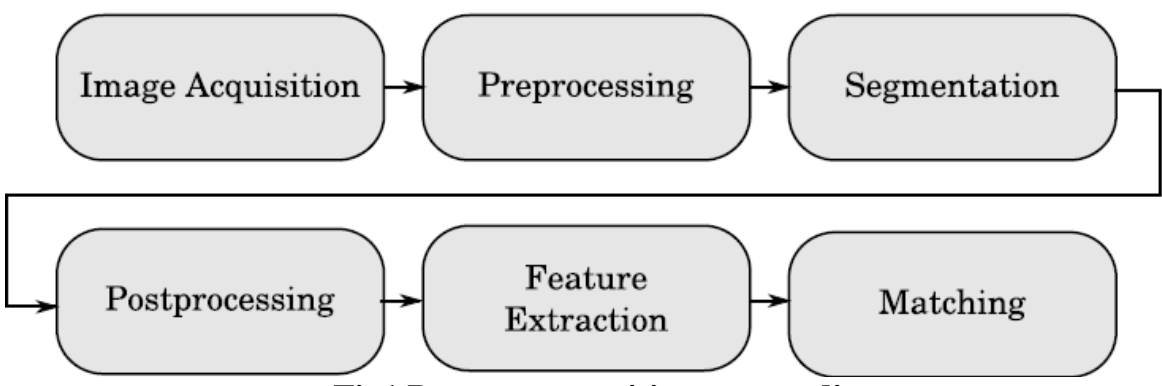

Fig1 Pattern recognition system diagram

\section{Image Acquisition \& Preprocessing}

The image of the hand is captured using an ordinary webcam that has been modified to only allow infrared light to reach the image sensor. After taking the image and before extraction of the vein pattern, 
preprocessing is applied to the image. The purpose of this step is to improve the imaging quality so that vein patterns can be more easily detected during the segmentation. This is done by first cropping the image to isolate the ROI and then applying filters to reduce noise and enhance the contrast.
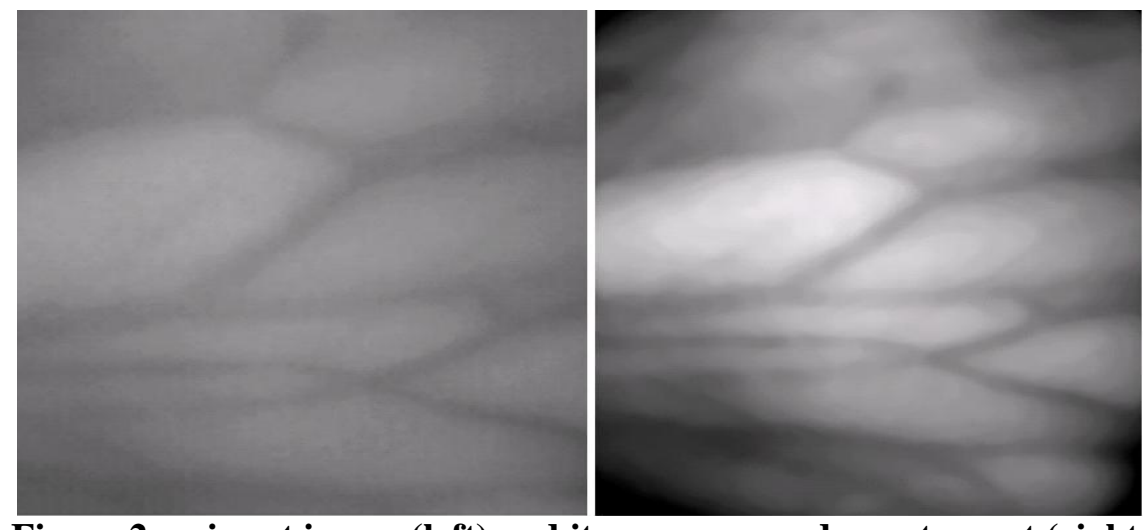

Figure 2 an input image (left) and its preprocessed counterpart (right)

\section{Segmentation \& Post-Processing}

Once the noise has been reduced and the contrast enhanced segmentation permits to separate the vein pattern from the background. Indeed the vein pattern is located and isolated from the rest of the image thus binarizing it. This is the most crucial step in the entire recognition process. If the veins are not properly detected the risk of errors increases greatly. Thus the chosen method plays a big role in the overall performance of the system. The output image of the segmentation step is a binary image with some unwanted information such as noise shadows and faint veins. Therefore it is not always a true representation of the actual vein pattern. The segmentation separates the vein pixels from the nonvein ones. Due to the importance of this step in the overall performance of the system, several segmentation algorithms have been implemented here including: Repeated Line Tracking (RLT) Laplacian of Gaussian (LoG) and Direction Based Vascular Pattern Extraction (DBVPE).Figure 3 shows four different preprocessed input images and their segmented counterparts obtained by these three segmentation methods.
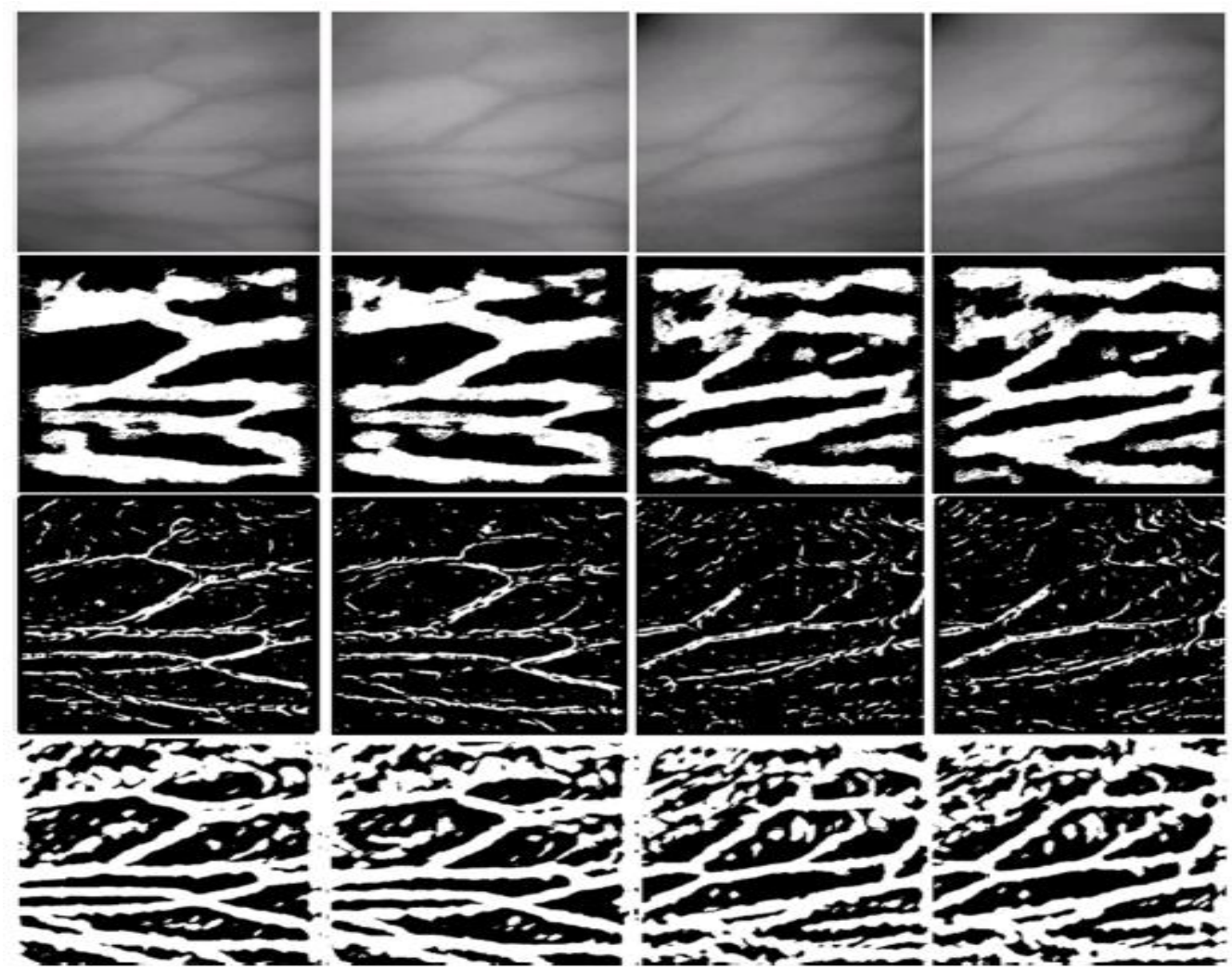

Fig3 from top, first row: four preprocessed input images and their segmented counterparts obtained by RLT (second row), LoG (third row), and DBVPE (last row) 


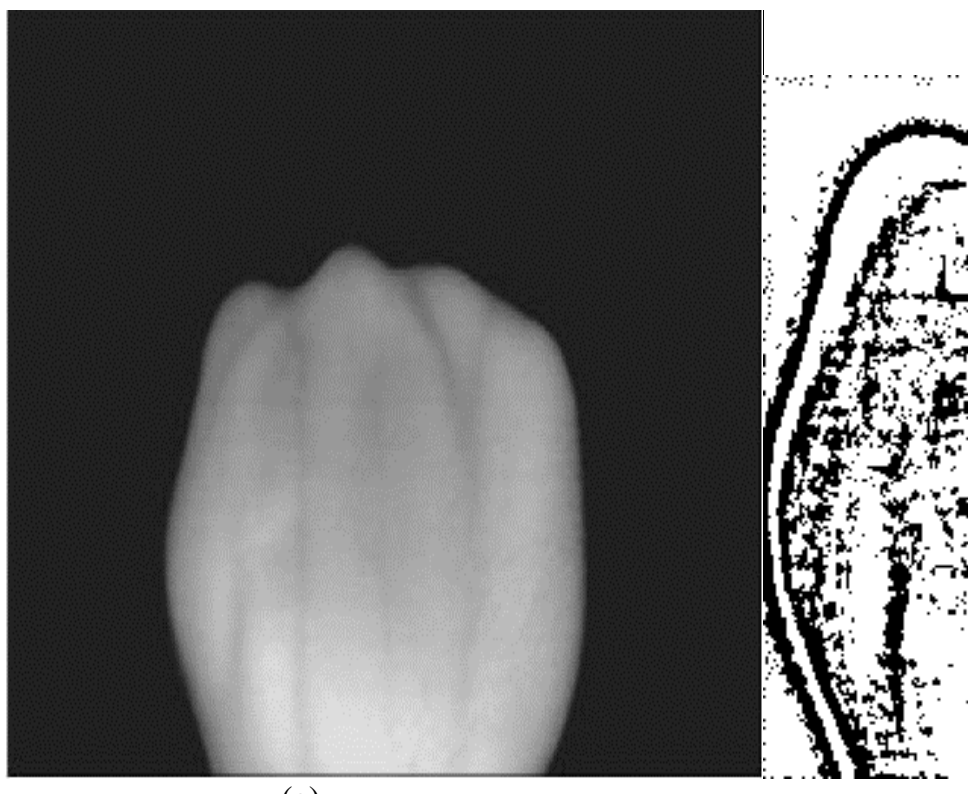

(a)

Fig4 (a) original image (b) segmented image

\section{Features Extraction \& Matching}

The feature extraction step aims to extract the actual features of the vein pattern from an image that they are going to be used for matching. If the image is an enrolled sample the features are saved in a database for later matching. Once the features are extracted they are compared with the ones in the database and based on that comparison a decision is taken. Thinning methods try to extract the skeleton of the vein pattern. The skeleton is a binary representation of the pattern with only one pixel wide. To extract the skeleton, the method explained in [2] has been used.

\section{CONCLUSION}

By modifying a very low-price webcam, the proposed system in this paper constructs a simple imaging setup for vein pattern recognition. The processing algorithm of the system composes different steps including: preprocessing segmentation, post-processing, feature extraction and finally recognition. Adaptive local thresholding using direction based vascular pattern extraction has been used for segmentation and a modified version of Hausdorff distance for the recognition. The experimental results of the system show that the system can achieve a zero false acceptance rate while its true acceptance rate is acceptably high. This is indeed the goal of the system as a lower true acceptance rate is less risky than a higher false acceptance rate. Because a person who is wrongly rejected can simply try again whiling the risk of accepting an imposter can be crucial.

\section{REFERENCES}

[1] A. K. Jain, P. Flynn, and A. A. Ross, Handbook of Biometrics. Springer, 2008, pp. 253\{270

[2] L. Lam, S. Lee, and C. Suen, "Thinning methodologies-a comprehensive survey," IEEE Transactions on Pattern Analysis and Machine Intelligence, vol. 14, no. 9, pp. 869-879, September 2002.

[3] S. K. Im, H. S. Choi, and S.-W. Kim., "A direction-based vascular pattern extraction algorithm for hand vascular pattern verication," ETRI journal, vol. 5, no. 2, pp.101-108, 2003.

[4] L. Wang, G. Leedham, and D. S.-Y. Cho, "Minutiae feature analysis for infrared hand vein pattern biometrics," Pattern Recognition, vol. 41, pp.920-929, 2008

[5] L. Wang and G. Leedham, "A thermal hand vein pattern verification system,” Lecture Notes in Computer Science, pp. 58-65, 2005 